\title{
Monotone Method for Nonlinear First-order Hyperbolic Initial-boundary Value Problems of Moving Boundary
}

\author{
Shaohua Wu, Di Chi* \\ School of Mathematics and Statistics, Wuhan University, Wuhan, China \\ Email address: \\ 13507142817@163.com (ShaohuaWu),dchiwhu@whu.edu.cn (Di Chi) \\ ${ }^{*}$ Corresponding author
}

To cite this article:

Shaohua Wu, Di Chi. Monotone Method for Nonlinear First-order Hyperbolic Initial-boundary Value Problems of Moving Boundary. American Journal of Applied Mathematics. Vol. 7, No. 3, 2019, pp. 80-89. doi: 10.11648/j.ajam.20190703.12

Received: June 21, 2019; Accepted: August 12, 2019; Published: August 30, 2019

\begin{abstract}
Moving boundary problems arise in many important applications to biology and chemistry. Comparing to the fixed boundary problem, moving boundary problem is more reasonable. To the best of our knowledge, there's few results on the moving boundary for nonlinear first-order hyperbolic initial-boundary value problems. In the present paper, we mainly clarify the problem and show the existence and uniqueness of the solution for such kind of problems. We take a classical transform to straighten the moving boundary and develop a monotone approximation, based on upper and lower solutions technique, for solving a class of first-order hyperbolic initial-boundary value problems of moving boundary. Such an approximation results in the existence and uniqueness of the solution for the problem. The idea behind such a method is to replace the actual solution in all the nonlinear and nonlocal terms with some previous guess for the solution, then solve the resulting linear model to obtain a new guess for the solution. Iteration of such a procedure yields the solution of the original problem upon passage to the limit. A novelty of such a technique is that an explicit solution representation for each of these iterates is obtained, and hence an efficient numerical scheme can be developed. The key step is a comparison principle between consecutive guesses.
\end{abstract}

Keywords: Hyperbolic IBVP, Moving Boundary, Upper-lower Solutions, Monotone Approximation

\section{Introduction}

Moving boundary problems deal with solving partial differential equations (PDEs) in a domain, a part of whose boundary is unknown in advance; that portion of the boundary is called a moving boundary. In addition to the standard boundary conditions that are needed in order to solve the PDEs, an additional condition must be imposed at the moving boundary. One then seeks to determine both the moving boundary and the solution of the differential equations. Recent decades moving boundary problems occur in such varied subjects as hydrology, heat flow, metallurgy,

$$
\left\{\begin{array}{l}
u_{t}+(V(x) u)_{x}=F(x, t, u, P(u(\cdot, t))), \\
V(0) u(0, t)=\int_{0}^{h(t)} \beta(x) u(x, t) d x, \\
u(x, 0)=u_{0}(x), \\
h^{\prime}(t)=V(h(t)),
\end{array}\right.
$$

molecular diffusion, flame propagation, steel and glass production, and oil drilling and mathematical finance [1-3].

Over the past several years, many authors have successfully applied the monotone method to nonlinear differential equations [4-6]. Based on the comparison principle, such a method involves the construction of monotone sequences of upper and lower solutions that converge uniformly to the solution of the problem being considered [7].

Our main objective here is to extend this method to the following first-order hyperbolic initial-boundary value problem of moving boundary

$$
\begin{aligned}
& x \in[0, h(t)], t \in[0, T], \\
& t \in[0, T], \\
& x \in[0, b], \\
& t \in[0, T],
\end{aligned}
$$


where $P(u(\cdot, t))=\int_{0}^{h(t)} u(y, t) d y$ and $x=h(t)$ is an unknown function which represents the free boundary.

The fixed boundary problem is defined by

$$
\begin{cases}u_{t}+(V(x) u)_{x}=F(x, t, u, P(u(\cdot, t))), & x \in[0, L], t \in[0, T], \\ V(0) u(0, t)=\int_{0}^{L} \beta(x) u(x, t) d x, & t \in[0, T], \\ u(x, 0)=u_{0}(x), & x \in[0, L],\end{cases}
$$

where $P(u(\cdot, t))=\int_{0}^{L} u(y, t) d y$.

The above problem arises in applied science. For example, in the case $F(x, t, u, P(u(\cdot, t)))=-m(x, t, P(u(\cdot, t))) u$, problem (2) describes the evolution of a size-structured population where individuals are competing for common resources $(\mathrm{m}$ represents the mortality rate which depends on the total population size P). For the size-structured population model, $\mathrm{u}$ is an unknown function which represents the density of individuals at times $t, V$ and $\beta$ denote the individuals' growth and reproduction rates, respectively. In practice, it is expected that such competition between individuals takes effect if the population level reaches high density, i.e., $m_{P}=0$ if $P \leq P_{0}$, and $m_{P}>0$ if $P>P_{0}$ [8-10].

Comparing to the fixed boundary problem in [11], moving boundary problem is more reasonable. The range of $\mathrm{x}$ is occupied in a fixed domain in standard literatures. In other words, the biggest size of the individual is fixed. However, the real phenomenon does not obey the law. As time goes on, the biggest size of the individual maybe change due to the environment around them. For example, $x$ represents the age of the individual. If the external environment is better, the maximum age is bigger.

To the best of our knowledge, there's few results on the moving boundary for nonlinear first-order hyperbolic initialboundary value problems. Our main goal in this paper is to clarify the problem and show the existence and uniqueness of the solution for such kind of problems by using monotone method.

This paper is arranged as follows. In section 2, we present the vital conditions and the main result of the paper. In section 3.1, we take a classical transform to straighten the moving boundary. A comparison result is discussed for the problem. Using upper and lower solution method, we establish the existence of the solution for such kind of the problem. In addition, the uniqueness of the solution is discussed in the following section 3.2.

\section{Main Result}

In this section, we state our main result. To state our theorem, we first impose the following hypotheses on the parameters in problem (1).

(C1) $V \in C^{1}([0, L]), V>0$ on $[0, L)$, and $V(\lambda x)>\lambda V(x)$ for any $\lambda>0$.

(C2) $\beta \in C([0, L])$ and $\beta \geq 0$ in $[0, L]$.

(C3) $\lim _{x \rightarrow b^{-}}\left(V u_{0}\right)(x)=0$, and $u_{0}$ satisfies the capability condition $V(0) u_{0}(x)=\int_{0}^{b} \beta(x) u_{0}(x) d x$.

(C4) $F \in C^{1}([0, L] \times[0, T] \times R \times R)$ and $F_{p} \leq 0$.
Our main result is as follows.

Theorem 2.1. Assume that (C1) - (C4) hold, If

$$
u_{0} \in H^{1}((0, b)) \text {, }
$$

Where $0<b<L$ is a constant, then there exist a unique solution $u(x, t)$ and a unique curve $x=h(t)$ which satisfy (1). Moreover, $x=h(t)$ is an increasing function and the solution is global in time.

Remark 2.1. The monotone of the function $x=h(t)$ is obvious, so we only show the existence and uniqueness of the solution for the model (1).

\section{The Proof of the Main Theorem}

In this section, we show the existence and uniqueness of the solution for nonlinear first-order hyperbolic initial boundary value problems (1), i.e. Theorem 2.1.

Before starting our main contents, we give simple description of the approaches. In order to achieve the goal, we shall processed as follows:

a) First, we show the existence and uniqueness of the moving boundary by the standard ODE theory;

b) Second, we straighten the free boundary and convert the problem to a fixed boundary problem. Then, we can show the existence of the solution $u(x, t)$ by comparison principle, monotone sequences and lower and upper solution methods;

c) Finally, we obtain the solution is indeed unique.

\subsection{Existence of the Solution}

\subsubsection{Existence and Uniqueness of the Moving Boundary}

Noticing $h^{\prime}(t)=V(h(t))$ and $V(x)$ is continuously differentiable with respect to $x$ and $t$, we get a unique continuous solution $h(t)$ by ODE standard theory [12].

\subsubsection{Straighten the Moving Boundary}

For that $h(t)$, we consider the problem

$\begin{cases}u_{t}+(V(x) u)_{x}=F(x, t, u, P(u(\cdot, t))), & x \in[0, h(t)], t \in[0, T], \\ V(0) u(0, t)=\int_{0}^{h(t)} \beta(x) u(x, t) d x, & t \in[0, T], \\ u(x, 0)=u_{0}(x), & x \in[0, b],\end{cases}$

Take the transform

$$
\xi=\frac{x}{h(t)}
$$

and set

$$
\tilde{u}(\xi, t)=u(h(t) \xi, t)=u(x, t) .
$$

A simple calculation shows

$$
\begin{gathered}
\tilde{u}_{t}=u_{t}+\xi h^{\prime}(t) u_{x}, \\
\tilde{u}_{\xi}=h(t) u_{x} .
\end{gathered}
$$

Thus $\tilde{u}$ satisfies the following equation 


$$
\begin{cases}\tilde{u}_{t}+\left(\frac{V(h(t) \xi)-\xi h \prime(t)}{h(t)} \tilde{u}\right)_{\xi}=F(h(t) \xi, t, \tilde{u}, \tilde{P}(\tilde{u}(\cdot, t)))-\frac{h^{\prime}(t)}{h(t)} \tilde{u}, & \xi \in[0,1], t \in[0, T], \\ V(0) \tilde{u}(0, t)=h(t) \int_{0}^{1} \beta(h(t) \xi) \tilde{u}(\xi, t) d \xi, & t \in[0, T], \\ \tilde{u}(\xi, 0)=u_{0}(b \xi), & \xi \in[0,1] .\end{cases}
$$

Where $\tilde{P}(\tilde{u}(\cdot, t))=h(t) \int_{0}^{1} \tilde{u}(\xi, t) d \xi$. To solve the problem, we follow the similar argument $[13,14]$.

\subsubsection{Comparison Principle}

Let $D_{T}=(0, T) \times(0,1)$, and we introduce the following definition of a pair of coupled upper and lower solutions of problem (3).

Definition 3.1. A pair of functions $\tilde{u}(\xi, t)$ and $\tilde{v}(\xi, t)$ are called an upper and a lower solution of $(3)$ on $D_{T}$, respectively, if all the following hold:

1. $\tilde{u}, \tilde{v} \in C\left(D_{T}\right) \cap L^{\infty}\left(D_{T}\right)$;

2. $\tilde{u}(\xi, 0) \geq u_{0}(b \xi) \geq \tilde{v}(\xi, 0)$, in $[0,1]$;

For every $t \in(0, T)$ and every nonnegative $\varphi(\xi, t) \in C^{1}\left(\overline{D_{T}}\right)$, we have

$$
\begin{aligned}
& \int_{0}^{1} \tilde{u}(\xi, t) \varphi(\xi, t) d \xi \geq \int_{0}^{1} \tilde{u}(\xi, 0) \varphi(\xi, 0) d \xi \\
& \quad+\int_{0}^{t} \varphi(0, s) \int_{0}^{1} \beta(h(s) \xi) \tilde{u}(\xi, s) d \xi d s \\
& \quad+\int_{0}^{t} \int_{0}^{1}\left(\varphi_{s}(\xi, s)+\frac{V(h(s) \xi)-\xi h^{\prime}(s)}{h(s)} \varphi_{\xi}(\xi, s)\right) \tilde{u}(\xi, s) d \xi d s \\
& \quad+\int_{0}^{t} \int_{0}^{1}\left(F(h(s) \xi, s, \tilde{u}(\xi, s), \tilde{P}(\tilde{v}(\cdot, s)))-\frac{h^{\prime}(s)}{h(s)} \tilde{u}(\xi, s)\right) \varphi(\xi, s) d \xi d s
\end{aligned}
$$

and

$$
\begin{aligned}
& \int_{0}^{1} \tilde{v}(\xi, t) \varphi(\xi, t) d \xi \leq \int_{0}^{1} \tilde{v}(\xi, 0) \varphi(\xi, 0) d \xi \\
& \quad+\int_{0}^{t} \varphi(0, s) \int_{0}^{1} \beta(h(s) \xi) \tilde{v}(\xi, s) d \xi d s \\
& \quad+\int_{0}^{t} \int_{0}^{1}\left(\varphi_{s}(\xi, s)+\frac{V(h(s) \xi)-\xi h^{\prime}(s)}{h(s)} \varphi_{\xi}(\xi, s)\right) \tilde{v}(\xi, s) d \xi d s \\
& \quad+\int_{0}^{t} \int_{0}^{1}\left(F(h(s) \xi, s, \tilde{v}(\xi, s), \tilde{P}(\tilde{u}(\cdot, s)))-\frac{h^{\prime}(s)}{h(s)} \tilde{v}(\xi, s)\right) \varphi(\xi, s) d \xi d s .
\end{aligned}
$$

Definition 3.2. A function $\tilde{u}(\xi, t)$ is called a weak solution of (3) on $D_{T}$ if $\tilde{u}$ is not only an upper solution but also a lower solution of $(3)$ on $\mathrm{D}_{\mathrm{T}}$.

Theorem 3.1. Suppose that the assumptions in Theorem 2.1 hold. Let $\tilde{u}$ and $\tilde{v}$ be a nonnegative upper solution and a nonnegative lower solution of (3), respectively. Then $\tilde{u} \geq \tilde{v}$ in $\overline{D_{T}}$.

Proof. Let $w=\tilde{v}-\tilde{u}$, then $w$ satisfies

$$
w(\xi, 0)=\tilde{v}(\xi, 0)-\tilde{u}(\xi, 0) \leqslant 0 \text { in }[0,1]
$$

and

$$
\begin{aligned}
\int_{0}^{1} w & (\xi, t) \varphi(\xi, t) d \xi \leq \int_{0}^{1} w(\xi, 0) \varphi(\xi, 0) d \xi \\
& +\int_{0}^{t} \varphi(0, s) \int_{0}^{1} \beta(h(s) \xi) w(\xi, s) d \xi d s \\
& +\int_{0}^{t} \int_{0}^{1}\left(\varphi_{s}(\xi, s)+\frac{V(h(s) \xi)-\xi h(s)}{h(s)} \varphi_{\xi}(\xi, s)\right) w(\xi, s) d \xi d s \\
\quad & +\int_{0}^{t} \int_{0}^{1} \varphi(\xi, s) A(\xi, s) w(\xi, s) d \xi d s+\int_{0}^{t} \int_{0}^{1} \varphi(\xi, s) B(\xi, s) \tilde{P}(w(\cdot, s)) d \xi d s \\
& -\int_{0}^{t} \int_{0}^{1} \frac{h \prime(s)}{h(s)} w(\xi, s) \varphi(\xi, s) d \xi d s,
\end{aligned}
$$


by mean value theorem,

where $A(\xi, t)=F_{\tilde{u}}\left(\xi, t, \theta_{1}(\xi, t), \tilde{P}(\tilde{u}(\cdot, t))\right)$ with $\theta_{1}$ between $\tilde{v}$ and $\tilde{u}$, and $B(\xi, t)=-F_{\tilde{P}}\left(\xi, t, \tilde{u}(\xi, t), \theta_{2}(t)\right)$ with $\theta_{2}$ between $\tilde{P}(\tilde{v}(\cdot, t))$ and $\tilde{P}(\tilde{u}(\cdot, t))$.

Let $\varphi(\xi, t)=e^{\lambda t} \psi(\xi, t)$ where $\psi \in C^{1}\left(\overline{D_{T}}\right)$ and $\lambda(\geq 0)$ is chosen so that $\lambda+F_{\widetilde{u}}-\frac{h^{\prime}(t)}{h(t)} \geq 0$, Then we find

$$
\begin{aligned}
& e^{\lambda t} \int_{0}^{1} w(\xi, t) \psi(\xi, t) d \xi \leq \int_{0}^{1} w(\xi, 0) \psi(\xi, 0) d \xi \\
& \quad+\int_{0}^{t} e^{\lambda s} \psi(0, s) \int_{0}^{1} \beta(h(s) \xi) w(\xi, s) d \xi d s \\
& \quad+\int_{0}^{t} \int_{0}^{1}\left(\psi_{s}(\xi, s)+\frac{V(h(s) \xi)-\xi h(s)}{h(s)} \psi_{\xi}(\xi, s)\right) e^{\lambda s} w(\xi, s) d \xi d s \\
& \quad+\int_{0}^{t} \int_{0}^{1}\left(\lambda+F_{\tilde{u}}\left(\xi, t, \theta_{1}(\xi, t), \tilde{P}(\tilde{u}(\cdot, t))\right)-\frac{h^{\prime}(s)}{h(s)}\right) w(\xi, s) e^{\lambda s} \psi(\xi, s) d \xi d s \\
& \quad+\int_{0}^{t} \int_{0}^{1} e^{\lambda s} \psi(\xi, s) B(\xi, t) \tilde{P}(w(\cdot, s)) d \xi d s .
\end{aligned}
$$

To simplify the above inequality, we now set up a backward problem as follows:

$$
\begin{cases}\psi_{s}(\xi, s)+\frac{V(h(s) \xi)-\xi h \prime(s)}{h(s)} \psi_{\xi}(\xi, s)=0, & 0<s<t, 0<\xi<1, \\ \psi(1, s)=0, & 0<s<t \\ \psi(\xi, t)=\zeta(\xi), & 0 \leq \xi \leq 1 .\end{cases}
$$

Here $\zeta(\xi) \in C_{0}^{\infty}(0,1), 0 \leq \zeta \leq 1$. The existence of $\psi \in C^{1}\left(\overline{D_{T}}\right)$ follows from the fact that by the variable change $\tau=t-s$, and let $\tilde{\psi}(\xi, \tau)=\psi(\xi, s)$. Then (7) can be written as

$$
\begin{cases}\tilde{\psi}_{\tau}(\xi, \tau)-\frac{V(h(t-\tau) \xi)+\xi h^{\prime}(t-\tau)}{h(t-\tau)} \tilde{\psi}_{\xi}(\xi, \tau)=0, & 0<\tau<t, 0<\xi<1 \\ \tilde{\psi}(1, \tau)=0, & 0<\tau<t \\ \tilde{\psi}(\xi, 0)=\zeta(\xi), & 0 \leq \xi \leq 1\end{cases}
$$

And (8) can be solved by the characteristic method. Note that the initial and boundary values for $\psi$ imply that $0 \leq \psi \leq$ 1 on $D_{T}$.

Substituting such a $\psi$ in (6) yields

$$
\begin{aligned}
& \int_{0}^{1} w(\xi, t) \zeta(\xi) d \xi \\
\leq & \int_{0}^{1} w(\xi, 0)^{+} d \xi \\
+ & C_{1} \int_{0}^{t} \int_{0}^{1} w(\xi, s)^{+} d \xi d s
\end{aligned}
$$

where $w(\xi, t)^{+}=\max \{w(\xi, t), 0\}$ and

$$
C_{1}=\max _{\overline{D_{T}}}\left\{\beta(h(t) \xi)+\left(\lambda+F_{\widetilde{u}}-\frac{h^{\prime}(t)}{h(t)}\right)+h(t) B(\xi, t)\right\} .
$$

From the condition on initial data in (4), we have

$$
\int_{0}^{1} w(\xi, t) \zeta(\xi) d \xi \leq C_{1} \int_{0}^{t} \int_{0}^{1} w(\xi, s)^{+} d \xi d s .
$$

Since this inequality holds for every $\zeta \in C_{0}^{\infty}(0,1)$ with $0 \leq \zeta \leq 1$, we can choose a sequence $\left\{\zeta_{n}\right\}_{n=1}^{\infty}$ on $(0,1)$ converging to

$$
\chi= \begin{cases}1, & w(\xi, t)>0 \\ 0, & \text { otherwise }\end{cases}
$$

Consequently, we find that

$$
\int_{0}^{1} w(\xi, t)^{+} d \xi \leq C_{1} \int_{0}^{t} \int_{0}^{1} w(\xi, s)^{+} d \xi d s,
$$

which by Gronwall's inequality leads to

$$
\int_{0}^{1} w(\xi, t)^{+} d \xi=0 .
$$

Thus, the proof is completed.

\subsubsection{Monotone Sequences and Existence of Solutions}

Now, we assume that $F(h(t) \xi, t, \tilde{u}, \tilde{P}(\tilde{v}(\cdot, t)))=$ $-m(h(t) \xi, t, \tilde{P}(\tilde{v}(\cdot, t))) \tilde{u}$, then we construct a pair of lower and upper solutions of $(3)$.

Let $\underline{u}^{0}(\xi, t)=0$ and $\bar{u}^{0}(\xi, t)=\delta e^{\sigma t} e^{-\gamma \xi}$, where $\delta, \sigma, \gamma$ are some determinate constants. Then it can be easily shown that $\underline{u}^{0}$ and $\bar{u}^{0}$ are a pair of coupled lower and upper solutions of $(3)$ on $[0,1] \times[0, T]$.

Actually, it is easily seen that $\underline{u}^{0}$ is a lower solution of (3). The task is now to show that is $\bar{u}^{0}$ an upper solution of (3).

1. $\bar{u}^{0} \in C\left(D_{T}\right) \cap L^{\infty}\left(D_{T}\right)$

2. $u_{0}(b \xi) \leq \delta e^{-\gamma} \leq \bar{u}^{0}(\xi, 0)$, according to the choice of the parameters $\delta$ and $\gamma$ in the following.

3. Notice

$$
\bar{u}_{t}^{0}(\xi, t)=\sigma \bar{u}^{0}(\xi, t), \quad \bar{u}_{\xi}^{0}(\xi, t)=-\gamma \bar{u}^{0}(\xi, t) .
$$


We have

$$
\begin{aligned}
& \int_{0}^{t} \varphi(0, s) \int_{0}^{1} \beta(h(s) \xi) \bar{u}^{0}(\xi, s) d \xi d s \\
= & \int_{0}^{t} \int_{0}^{1} \frac{\beta(h(s) \xi) h(s)}{V(0)} e^{-\gamma \xi} d \xi \frac{V(0)}{h(s)} \bar{u}^{0}(0, s) \varphi(0, s) d s .
\end{aligned}
$$

Choose a constant $\gamma>0$ large enough such that

$$
\max _{\overline{D_{T}}} \frac{\beta(h(s) \xi) h(s)}{V(0)} \leq \frac{\gamma}{2}
$$

and then choose $\delta>0$ large enough such that

$$
\left\|u_{0}\right\|_{L^{\infty}} \leq \delta e^{-\gamma} .
$$

Hence, it holds that

$$
\begin{gathered}
\int_{0}^{t} \varphi(0, s) \int_{0}^{1} \beta(h(s) \xi) \bar{u}^{0}(\xi, s) d \xi d s \\
\leq \int_{0}^{t} \frac{V(0)}{h(s)} \bar{u}^{0}(0, s) \varphi(0, s) d s .
\end{gathered}
$$

We have

$$
\begin{aligned}
& \int_{0}^{t} \int_{0}^{1} \varphi_{s}(\xi, s) \bar{u}^{0}(\xi, s) d \xi d s \\
&= \int_{0}^{1} \varphi(\xi, t) \bar{u}^{0}(\xi, t) d \xi-\int_{0}^{1} \varphi(\xi, 0) \bar{u}^{0}(\xi, 0) d \xi \\
&-\sigma \int_{0}^{t} \int_{0}^{1} \varphi(\xi, s) \bar{u}^{0}(\xi, s) d \xi d s,
\end{aligned}
$$

and

$$
\begin{gathered}
\int_{0}^{t} \int_{0}^{1} \frac{V(h(s) \xi)-\xi h^{\prime}(s)}{h(s)} \varphi_{\xi}(\xi, s) \bar{u}^{0}(\xi, s) d \xi d s \\
=\int_{0}^{t} \frac{V(h(s))-h^{\prime}(s)}{h(s)} \varphi(1, s) \bar{u}^{0}(1, s) d s-\int_{0}^{t} \frac{V(0)}{h(s)} \varphi(0, s) \bar{u}^{0}(0, s) d s \\
-\int_{0}^{t} \int_{0}^{1} \frac{V_{x}(h(s) \xi) h(s)-h^{\prime}(s)}{h(s)} \varphi(\xi, s) \bar{u}^{0}(\xi, s) d \xi d s \\
+\gamma \int_{0}^{t} \int_{0}^{1} \frac{V(h(s) \xi)-\xi h^{\prime}(s)}{h(s)} \varphi(\xi, s) \bar{u}^{0}(\xi, s) d \xi d s .
\end{gathered}
$$

by integrating by parts. Then, it holds that

$$
\begin{aligned}
& \int_{0}^{1} \bar{u}^{0}(\xi, 0) \varphi(\xi, 0) d \xi+\int_{0}^{t} \varphi(0, s) \int_{0}^{1} \beta(h(s) \xi) \bar{u}^{0}(\xi, s) d \xi d s \\
& +\int_{0}^{t} \int_{0}^{1}\left(\varphi_{s}(\xi, s)+\frac{V(h(s) \xi)-\xi h^{\prime}(s)}{h(s)} \varphi_{\xi}(\xi, s)\right) \bar{u}^{0}(\xi, s) d \xi d s \\
& -\int_{0}^{t} \int_{0}^{1}\left(m\left(h(s) \xi, s, \tilde{P}\left(\underline{u}^{0}(\cdot, s)\right)\right)+\frac{h^{\prime}(s)}{h(s)}\right) \bar{u}^{0}(\xi, s) \varphi(\xi, s) d \xi d s \\
\leq & \int_{0}^{1} \bar{u}^{0}(\xi, t) \varphi(\xi, t) d \xi \\
& +\int_{0}^{t} \int_{0}^{1}\left(-\sigma-V_{x}(h(s) \xi)+\gamma \frac{V(h(s) \xi)-\xi h^{\prime}(s)}{h(s)}\right) \times \varphi(\xi, s) \bar{u}^{0}(\xi, s) d \xi d s,
\end{aligned}
$$

by the fourth equation of (1). Choose $\sigma>0$ large enough such that

$$
\sigma \geq \max _{\overline{D_{T}}}\left|V_{x}(h(s) \xi)\right|+\gamma \max _{\overline{D_{T}}} \frac{V(h(s) \xi)-\xi h^{\prime}(s)}{h(s)} .
$$

Then $\bar{u}^{0}$ is an upper solution of (3).

Under hypothesis (C4), we can choose a positive constant $\mathrm{M}$ such that $F_{\widetilde{u}}(h(t) \xi, t, \tilde{u}, \tilde{P}(\tilde{v}(\cdot, t)))+M \geq 0$ for $(\xi, t) \in \overline{D_{T}}$, we then define two sequences $\left\{\underline{u}^{k}\right\}_{k=0}^{\infty}$ and $\left\{\bar{u}^{k}\right\}_{k=0}^{\infty}$ as follows.

For $k=1,2, \cdots$,

$$
\begin{cases}\underline{u}_{t}^{k}+\left(\frac{V(h(t) \xi)-\xi h^{\prime}(t)}{h(t)} \underline{u}^{k}\right)_{\xi}=F\left(h(t) \xi, t, \underline{u}^{k-1}, \tilde{P}\left(\bar{u}^{k-1}(\cdot, t)\right)\right)-\frac{h^{\prime}(t)}{h(t)} \underline{u}^{k}-M\left(\underline{u}^{k}-\underline{u}^{k-1}\right), & \xi \in[0,1], t \in[0, T], \\ V(0) \underline{u}^{k}(0, t)=h(t) \int_{0}^{1} \beta(h(t) \xi) \underline{u}^{k-1}(\xi, t) d \xi & t \in[0, T] \\ \underline{u}^{k}(\xi, 0)=u_{0}(b \xi), & \xi \in[0,1]\end{cases}
$$


and

$$
\begin{cases}\bar{u}_{t}^{k}+\left(\frac{V(h(t) \xi)-\xi h^{\prime}(t)}{h(t)} \bar{u}^{k}\right)_{\xi}=F\left(h(t) \xi, t, \bar{u}^{k-1}, \tilde{P}\left(\underline{u}^{k-1}(\cdot, t)\right)\right)-\frac{h^{\prime}(t)}{h(t)} \bar{u}^{k}-M\left(\bar{u}^{k}-\bar{u}^{k-1}\right), & \xi \in[0,1], t \in[0, T], \\ V(0) \bar{u}^{k}(0, t)=h(t) \int_{0}^{1} \beta(h(t) \xi) \bar{u}^{k-1}(\xi, t) d \xi, & t \in[0, T], \\ \bar{u}^{k}(\xi, 0)=u_{0}(b \xi), & \xi \in[0,1],\end{cases}
$$

Since $\underline{u}^{0}(\xi, t)$ and $\bar{u}^{0}(\xi, t)$ are continuously differentiable in $t$, the existence of the solutions for the problems (9) and (10) is guaranteed by theorem 1.11 in [15], In fact, these systems can be solved using the method of characteristics. Consider the equation for the characteristic curves given by

$$
\left\{\begin{array}{l}
\frac{d}{d s} t(s)=1 \\
\frac{d}{d s} \xi(s)=\frac{V(h(t(s)) \xi(s))-\xi(s) h^{\prime}(t(s))}{h(t(s))} .
\end{array}\right.
$$

The solution $\underline{u}^{k}$ of (9) along the characteristic curve $(\xi(s), t(s))$ satisfies the following equation

$$
\frac{d}{d s} \underline{u}^{k}(s)=-V_{x}(h(t(s)) \xi(s)) \underline{u}^{k}(s)+F\left(h(t(s)) \xi(s), t(s), \underline{u}^{k-1}(s), \tilde{P}\left(\bar{u}^{k-1}(\cdot, t(s))\right)\right)-M\left(\underline{u}^{k}(s)-\underline{u}^{k-1}(s)\right) .
$$

Parametrizing the characteristic curves with the variable $t$, then a characteristic curve passing through $(\hat{\xi}, \hat{t})$ is given by $(t, X(t ; \hat{\xi}, \hat{t}))$ where $X$ satisfies

$$
\frac{d}{d t} X(t ; \hat{\xi}, \hat{t})=\frac{V(h(t) X(t ; \hat{\xi}, \hat{t}))-X(t ; \hat{\xi}, \hat{t}) h^{\prime}(t)}{h(t)}
$$

and $X(\hat{t} ; \hat{\xi}, \hat{t})=\hat{\xi}$. From (C1), it follows that the function $X$ is strictly increasing. Hence, a unique inverse function $\tau(\xi ; \hat{t}, \hat{\xi})$ exists. Now we define $G(\xi)=\tau(\xi ; 0,0)$ where $(G(\xi), \xi)$ represents the characteristic curve passing through $(0,0)$ which divides the $(\xi, t)$-plane into two parts. Then for any point $(\xi, t)$ with $t \leq G(\xi)$, the solution $\underline{u}^{k}(\xi, t)$ is determined through the initial condition by

$$
\begin{aligned}
& \underline{u}^{k}(\xi, t)=u_{0}(b X(0 ; \xi, t)) B(t ; 0)+\int_{0}^{t} B(t ; s) \\
& \quad \times\left[F\left(h(s) X(s ; \xi, t), s, \underline{u}^{k-1}(X(s ; \xi, t), s), \tilde{P}\left(\bar{u}^{k-1}(\cdot, s)\right)\right)\right. \\
& \left.\quad+M \underline{u}^{k-1}(X(s ; \xi, t), s)\right] d s
\end{aligned}
$$

and for any point $(\xi, t)$ with $t>G(\xi)$ the solution is determined via the boundary condition by

$$
\begin{aligned}
& \underline{u}^{k}(\xi, t)=R^{k-1}(\tau(0 ; \xi, t)) B(t ; \tau(0 ; \xi, t))+\int_{\tau(0 ; \xi, t)}^{t} B(t ; s) \\
& \quad \times\left[F\left(h(s) X(s ; \xi, t), s, \underline{u}^{k-1}(X(s ; \xi, t), s), \tilde{P}\left(\bar{u}^{k-1}(\cdot, s)\right)\right)\right. \\
& \left.\quad+M \underline{u}^{k-1}(X(s ; \xi, t), s)\right] d s
\end{aligned}
$$

where

$$
B(t ; s)=\exp \left(-M(t-s)-\int_{s}^{t} V_{x}(h(s) X(s ; \xi, t)) d \varsigma\right)
$$

and

$$
\underline{R}^{k-1}(t)=\frac{h(t)}{V(0)} \int_{0}^{1} \beta(h(t) \xi) \underline{u}^{k-1}(\xi, t) d \xi
$$

Similarly, for any point $(\xi, t)$ with $t \leq G(\xi)$, the solution $\bar{u}^{k}(\xi, t)$ is determined through the initial condition by

$$
\begin{aligned}
& \bar{u}^{k}(\xi, t)=u_{0}(b X(0 ; \xi, t)) B(t ; 0)+\int_{0}^{t} B(t ; s) \\
& \quad \times\left[F\left(h(s) X(s ; \xi, t), s, \bar{u}^{k-1}(X(s ; \xi, t), s), \tilde{P}\left(\underline{u}^{k-1}(\cdot, s)\right)\right)\right. \\
& \left.\quad+M \bar{u}^{k-1}(X(s ; \xi, t), s)\right] d s
\end{aligned}
$$

and for any point $(\xi, t)$ with $t>G(\xi)$ the solution is determined via the boundary condition by

$$
\begin{aligned}
& \bar{u}^{k}(\xi, t)=R^{k-1}(\tau(0 ; \xi, t)) B(t ; \tau(0 ; \xi, t))+\int_{\tau(0 ; \xi, t)}^{t} B(t ; s) \\
& \quad \times\left[F\left(h(s) X(s ; \xi, t), s, \bar{u}^{k-1}(X(s ; \xi, t), s), \tilde{P}\left(\underline{u}^{k-1}(\cdot, s)\right)\right)\right. \\
& \left.\quad+M \bar{u}^{k-1}(X(s ; \xi, t), s)\right] d s
\end{aligned}
$$

where

$$
B(t ; s)=\exp \left(-M(t-s)-\int_{s}^{t} V_{x}(h(\varsigma) X(\varsigma ; \xi, t)) d \varsigma\right)
$$

and

$$
\bar{R}^{k-1}(t)=\frac{h(t)}{V(0)} \int_{0}^{1} \beta(h(t) \xi) \bar{u}^{k-1}(\xi, t) d \xi .
$$

Next we show that the sequences $\left\{\underline{u}^{k}\right\}_{k=0}^{\infty}$ and $\left\{\bar{u}^{k}\right\}_{k=0}^{\infty}$ are monotone by induction. The procedure of induction is as follows.

Step 1: Initial hypothesis of the induction;

We first let $w=\underline{u}^{0}-\underline{u}^{1}$. Then $w$ satisfies 


$$
\begin{aligned}
& \int_{0}^{1} w(\xi, t) \varphi(\xi, t) d \xi \leq \int_{0}^{1} w(\xi, 0) \varphi(\xi, 0) d \xi \\
& +\int_{0}^{t} \varphi(0, s) \int_{0}^{1} \beta(h(s) \xi) w(\xi, s) d \xi d s \\
& +\int_{0}^{t} \int_{0}^{1}\left(\varphi_{s}(\xi, s)+\frac{V(h(s) \xi)-\xi h^{\prime}(s)}{h(s)} \varphi_{\xi}(\xi, s)\right) w(\xi, s) d \xi d s \\
& -\int_{0}^{t} \int_{0}^{1} M w(\xi, s) \varphi(\xi, s) d \xi d s-\int_{0}^{t} \int_{0}^{1} \frac{h^{\prime}(s)}{h(s)} w(\xi, s) \varphi(\xi, s) d \xi d s .
\end{aligned}
$$

Similar to the proof of Theorem 3.1, we can get $w \leq 0$ which implies $\underline{u}^{0} \leq \underline{u}^{1}$. Similarly, it can be seen that $\bar{u}^{0} \geq \bar{u}^{1}$.

Second, we let $w=\underline{u}^{1}-\bar{u}^{0}$, since $\underline{u}^{0} \leq \underline{u}^{1}, w$ satisfies the same inequality with the same $A(\xi, t)=-M$ and $B(\xi, t)=0$ in (5). Thus, by the comparison principle, $\underline{u}^{1} \leq \bar{u}^{0}$. Similarly,

it can be seen that $\underline{u}^{0} \leq \bar{u}^{1}$. We now claim that $\underline{u}^{1}$ and $\bar{u}^{1}$ are a lower and an upper solution of (3), respectively. On the one hand, by(C4) and $\underline{u}^{0} \leq \underline{u}^{1} \leq \bar{u}^{0}$, the right-hand side of the equation in (9) satisfies

$$
\begin{aligned}
& F\left(h(t) \xi, t, \underline{u}^{0}, \tilde{P}\left(\bar{u}^{0}(\cdot, t)\right)\right)-M\left(\underline{u}^{1}-\underline{u}^{0}\right) \\
& =-\left[F_{u}\left(h(t) \xi, t, \theta_{0}, \tilde{P}\left(\bar{u}^{0}(\cdot, t)\right)\right)+M\right]\left(\underline{u}^{1}-\underline{u}^{0}\right)+F\left(h(t) \xi, t, \underline{u}^{1}, \tilde{P}\left(\bar{u}^{0}(\cdot, t)\right)\right) \\
& \leq F\left(h(t) \xi, t, \underline{u}^{1}, \tilde{P}\left(\bar{u}^{1}(\cdot, t)\right)\right)
\end{aligned}
$$

with $\underline{u}^{0} \leq \theta_{0} \leq \bar{u}^{0}$.

On the other hand, by (C4) and $\underline{u}^{0} \geq \bar{u}^{1} \geq \bar{u}^{0}$, the right-hand side of the equation in (10) satisfies

$$
\begin{aligned}
& F\left(h(t) \xi, t, \bar{u}^{0}, \tilde{P}\left(\underline{u}^{0}(\cdot, t)\right)\right)-M\left(\bar{u}^{1}-\bar{u}^{0}\right) \\
& =\left[F_{u}\left(h(t) \xi, t, \theta_{1}, \tilde{P}\left(\underline{u}^{0}(\cdot, t)\right)\right)+M\right]\left(\bar{u}^{0}-\bar{u}^{1}\right)+F\left(h(t) \xi, t, \bar{u}^{1}, \tilde{P}\left(\underline{u}^{0}(\cdot, t)\right)\right) \\
& \left.\geq F\left(h(t) \xi, t, \bar{u}^{1}, \tilde{P} \underline{u}^{1}(\cdot, t)\right)\right)
\end{aligned}
$$

with $\underline{u}^{0} \leq \theta_{1} \leq \bar{u}^{0}$.

Step 2: Hypothesis and claim of the induction;

We then assume that for some $k>1, \underline{u}^{k}$ and $\bar{u}^{k}$ are a lower and an upper solution of (3), respectively. By similar reasoning, we can show that $\underline{u}^{k} \leq \underline{u}^{k+1} \leq \bar{u}^{k+1} \leq \bar{u}^{k}$ and that $\underline{u}^{k+1}$ and $\bar{u}^{k+1}$ are also a lower and an upper solution of (3), respectively.

Thus, by induction, we obtain two monotone sequences that satisfy

$$
\underline{u}^{0} \leqslant \underline{u}^{1} \leqslant \cdots \leqslant \underline{u}^{k} \leqslant \bar{u}^{k} \leqslant \cdots \bar{u}^{1} \leqslant \bar{u}^{0} \text { in } \overline{D_{T}} .
$$

for each $k=0,1,2, \cdots$. Hence it follows from the monotonicity of the sequences $\left\{\underline{u}^{k}\right\}_{k=0}^{\infty}$ and $\left\{\bar{u}^{k}\right\}_{k=0}^{\infty}$, there exist functions $\underline{u}$ and $\bar{u}$ such that $\underline{u}^{k} \rightarrow \underline{\mathrm{u}}$ and $\bar{u}^{k} \rightarrow \bar{u}$ pointwise in $D_{T}$. Clearly $\underline{\mathrm{u}} \leq \bar{u}$ in $D_{T}$.

Upon establishing the monotonicity of the sequences, we now prove the sequences $\left\{\underline{u}^{k}\right\}_{k=0}^{\infty}$ and $\left\{\bar{u}^{k}\right\}_{k=0}^{\infty}$ converge uniformly along characteristic curves to a limit function $\tilde{u}$.

On the one hand, owing to $\underline{u}^{0} \leq \underline{u}^{k} \leq \bar{u}^{0}$ and the monotonicity of the sequence $\left\{\underline{u}^{k}\right\}$, we obtain by arguing as in [5], along the characteristic curve passing through $\left(\xi_{0}, 0\right)$, the solution

$$
\begin{aligned}
& \underline{u}^{k}\left(X\left(t ; \xi_{0}, 0\right), t\right)=u_{0}\left(b \xi_{0}\right) B(t ; 0)+\int_{0}^{t} B(t ; s) \\
& \quad \times\left[F\left(h(s) X\left(s ; \xi_{0}, 0\right), s, \underline{u}^{k-1}\left(X\left(s ; \xi_{0}, 0\right), s\right), \tilde{P}\left(\bar{u}^{k-1}(\cdot, s)\right)\right)\right. \\
& \left.\quad+M \underline{u}^{k-1}\left(X\left(s ; \xi_{0}, 0\right), s\right)\right] d s
\end{aligned}
$$

converges to

$$
\begin{aligned}
& \underline{\mathrm{u}}\left(X\left(t ; \xi_{0}, 0\right), t\right)=u_{0}\left(b \xi_{0}\right) B(t ; 0)+\int_{0}^{t} B(t ; s) \\
& \quad \times\left[F\left(h(s) X\left(s ; \xi_{0}, 0\right), s, \underline{\mathrm{u}}\left(X\left(s ; \xi_{0}, 0\right), s\right), \tilde{P}(\bar{u}(\cdot, s))\right)\right. \\
& \left.\quad+M \underline{\mathrm{u}}\left(X\left(s ; \xi_{0}, 0\right), s\right)\right] d s
\end{aligned}
$$

uniformly and monotonically for $0 \leq t \leq T$, where

$$
B(t ; s)=\exp \left(-M(t-s)-\int_{s}^{t} V_{x}(h(s) X(s ; \xi, t)) d s\right)
$$

On the other hand, along the characteristic curve passing through $\left(0, t_{0}\right)$, the solution

$$
\begin{aligned}
& \underline{u}^{k}\left(X\left(t ; 0, t_{0}\right), t\right)=R^{k-1}\left(t_{0}\right) B\left(t ; t_{0}\right)+\int_{t_{0}}^{t} B(t ; s) \\
& \quad \times\left[F\left(h(s) X\left(s ; 0, t_{0}\right), s, \underline{u}^{k-1}\left(X\left(s ; 0, t_{0}\right), s\right), \tilde{P}\left(\bar{u}^{k-1}(\cdot, s)\right)\right)\right. \\
& \left.\quad+M \underline{u}^{k-1}\left(X\left(s ; 0, t_{0}\right), s\right)\right] d s
\end{aligned}
$$

converges to 


$$
\begin{aligned}
& \underline{\mathrm{u}}\left(X\left(t ; 0, t_{0}, 0\right), t\right)=R\left(t_{0}\right) B\left(t ; t_{0}\right)+\int_{t_{0}}^{t} B(t ; s) \\
& \quad \times\left[F\left(h(s) X\left(s ; 0, t_{0}\right), s, \underline{\mathrm{u}}\left(X\left(s ; 0, t_{0}\right), s\right), \tilde{P}(\bar{u}(\cdot, s))\right)\right. \\
& \left.\quad+M \underline{\mathrm{u}}\left(X\left(s ; 0, t_{0}\right), s\right)\right] d s
\end{aligned}
$$

uniformly and monotonically for $0 \leq t \leq T$, where

$$
\underline{R}(t)=\frac{h(t)}{V(0)} \int_{0}^{1} \beta(h(t) \xi) \underline{u}(\xi, t) d \xi
$$

Thus, we have

$$
\underline{u}(\xi, t)=\left\{\begin{array}{cc}
u_{0}(b X(0 ; \xi, t)) B(t ; 0)+\int_{0}^{t} B(t ; s) & \\
\times[F(h(s) X(s ; \xi, t), s, \underline{u}(X(s ; \xi, t), s), \tilde{P}(\bar{u}(\cdot, s))) & \\
+M \underline{u}(X(s ; \xi, t), s)] d s, & t \leqslant G(\xi) ; \\
R(\tau(0 ; \xi, t)) B(t ; \tau(0 ; \xi, t))+\int_{\tau(0 ; \xi, t)}^{t} B(t ; s) & \\
\times[F(h(s) X(s ; \xi, t), s, \underline{u}(X(s ; \xi, t), s), \tilde{P}(\bar{u}(\cdot, s))) & \\
+M \underline{u}(X(s ; \xi, t), s)] d s, & t>G(\xi) .
\end{array}\right.
$$

Similarly, we have

$$
\bar{u}(\xi, t)=\left\{\begin{array}{cc}
u_{0}(b X(0 ; \xi, t)) B(t ; 0)+\int_{0}^{t} B(t ; s) & \\
\times[F(h(s) X(s ; \xi, t), s, \bar{u}(X(s ; \xi, t), s), \tilde{P}(\underline{u}(\cdot, s))) & \\
+M \bar{u}(X(s ; \xi, t), s)] d s, & t \leqslant G(\xi) ; \\
R(\tau(0 ; \xi, t)) B(t ; \tau(0 ; \xi, t))+\int_{\tau(0 ; \xi, t)}^{t} B(t ; s) & \\
\times[F(h(s) X(s ; \xi, t), s, \bar{u}(X(s ; \xi, t), s), \tilde{P}(\underline{u}(\cdot, s))) & \\
+M \bar{u}(X(s ; \xi, t), s)] d s, & t>G(\xi) .
\end{array}\right.
$$

where

$$
\bar{R}(t)=\frac{h(t)}{V(0)} \int_{0}^{1} \beta(h(t) \xi) \bar{u}(\xi, t) d \xi
$$

We now show that $\underline{\mathrm{u}}(\xi, t)=\bar{u}(\xi, t)$. Let $w=\bar{u}(\xi, t)-$ $\underline{u}(\xi, t)$. Since $\bar{u}(\xi, t) \geq \underline{\mathrm{u}}(\xi, t), w(\xi, t) \geq 0$ and $w(\xi, 0)=$ 0 . Hence $w$ satisfies

$$
\begin{aligned}
\int_{0}^{1} w(\xi, t) d \xi= & \int_{0}^{t} \int_{0}^{1} \beta(h(s) \xi) w(\xi, s) d \xi d s \\
& +\int_{0}^{t} \int_{0}^{1} A(\xi, s) w(\xi, s) d \xi d s \\
& +\int_{0}^{t} \int_{0}^{1} B(\xi, s) \tilde{P}(w(\cdot, s)) d \xi d s \\
& -\int_{0}^{t} \int_{0}^{1} \frac{h^{\prime}(s)}{h(s)} w(\xi, s) d \xi d s \\
\leq & C_{0} \int_{0}^{t} \int_{0}^{1} w(\xi, s) d \xi d s
\end{aligned}
$$

by choosing $\varphi(\xi, t)=1$ of (5), where

$$
C_{0}=\max _{\overline{D_{T}}}[\beta(h(t) \xi)+A(\xi, t)+h(t) B(\xi, t)] .
$$

Owing to Gronwall's inequality, we conclude $w(\xi, t)=0$ in $D_{T}$, i.e. $\bar{u}=\underline{u}$. Defining this common limit by u, we find that $\mathrm{u}$ satisfies the following. For any point $(\xi, t)$ with $t \leq G(\xi)$,

$$
\begin{aligned}
& u(\xi, t)=u_{0}(b X(0 ; \xi, t)) B(t ; 0)+\int_{0}^{t} B(t ; s) \\
& \quad \times[F(h(s) X(s ; \xi, t), s, u(X(s ; \xi, t), s), \tilde{P}(u(\cdot, s))) \\
& \quad+M u(X(s ; \xi, t), s)] d s
\end{aligned}
$$

and for any point $(\xi, t)$ with $t>G(\xi)$,

$$
\begin{aligned}
& u(\xi, t)=R(\tau(0 ; \xi, t)) B(t ; \tau(0 ; \xi, t))+\int_{\tau(0 ; \xi, t)}^{t} B(t ; s) \\
& \quad \times[F(h(s) X(s ; \xi, t), s, u(X(s ; \xi, t), s), \widetilde{P}(u(\cdot, s))) \\
& \quad+M u(X(s ; \xi, t), s)] d s
\end{aligned}
$$


Remark 3.1. We can also obtain that, along the characteristic curve passing through $\left(\xi_{0}, 0\right)$, the solution $\underline{u}^{k}\left(X\left(t ; \xi_{0}, 0\right), t\right)$ converges to $\underline{u}\left(X\left(t ; \xi_{0}, 0\right), t\right)$ uniformly and

$$
\begin{aligned}
& \underline{u}^{k+1}\left(X\left(t ; \xi_{0}, 0\right), t\right)-\underline{u}^{k}\left(X\left(t ; \xi_{0}, 0\right), t\right) \\
= & \left.\int_{0}^{t} B(t ; s) \times\left[Q^{k}(s)+M \underline{u}^{k}\left(X\left(s ; \xi_{0}, 0\right), s\right)\right] d s-\int_{0}^{t} B(t ; s) \times\left[Q^{k-1}(s)+M \underline{u}^{k-1}\left(X\left(s ; \xi_{0}, 0\right)\right), s\right)\right] d s \\
= & \int_{0}^{t} B(t ; s) \times\left[Q^{k}(s)-\left(Q^{k-1}(s)-M\left(\underline{u}^{k}\left(X\left(s ; \xi_{0}, 0\right), s\right)-\underline{u}^{k-1}\left(X\left(s ; \xi_{0}, 0\right), s\right)\right)\right)\right] d s \\
= & \int_{0}^{t} B(t ; s) \times\left[Q^{k}(s)-\left(\underline{u}_{t}^{k}+\left(\frac{V(h(t) \xi)-\xi h^{\prime}(t)}{h(t)} \underline{u}^{k}\right)_{\xi}+\frac{h^{\prime}(t)}{h(t)} \underline{u}^{k}\right)\right] d s \geq 0 .
\end{aligned}
$$

monotonically for $0 \leq t \leq T$, by using the Cauchy criterion of uniformly convergent of functions. Actually, we have

by comparison principle. From the monotonicity of the sequences $\left\{\underline{u}^{k}\right\}_{k=0}^{\infty}$ and $\left\{\bar{u}^{k}\right\}_{k=0}^{\infty}$, we get $\bar{u}^{k} \geq \underline{u}^{k}$ in $D_{T}$, and hence $\tilde{P}\left(\bar{u}^{k}(\cdot, t)\right) \geq \tilde{P}\left(\underline{u}^{k}(\cdot, t)\right)$ for $0 \leq t \leq T$, in view of the hypotheses (C4), we have $Q^{k}(s) \leq T^{k}(s)$, where

$$
\begin{aligned}
& Q^{k-1}(t)=F\left(h(t) X\left(t ; \xi_{0}, 0\right), t, \underline{u}^{k-1}\left(X\left(t ; \xi_{0}, 0\right), t\right), \tilde{P}\left(\bar{u}^{k-1}(\cdot, t)\right)\right), \\
& T^{k-1}(t)=F\left(h(t) X\left(t ; \xi_{0}, 0\right), t, \underline{u}^{k-1}\left(X\left(t ; \xi_{0}, 0\right), t\right), \tilde{P}\left(\underline{u}^{k-1}(\cdot, t)\right)\right),
\end{aligned}
$$

Then, we find that

$$
\left|\underline{u}^{k+1}\left(X\left(t ; \xi_{0}, 0\right), t\right)-\underline{u}^{k}\left(X\left(t ; \xi_{0}, 0\right), t\right)\right| \rightarrow 0, \quad \text { as } \quad k \rightarrow \infty .
$$

Proceeding in a similar manner, the uniform convergence can also be obtained analogously along the characteristic curve passing through $\left(0, t_{0}\right)$.

\subsection{Uniqueness of the Solution}

Using arguments in [16], we can establish $P(\tilde{u}(\cdot, t))$ is continuous. Hence, we claim $\tilde{u}$ is unique.

Actually, we suppose $\tilde{u}_{1}(\xi, t)$ and $\tilde{u}_{2}(\xi, t)$ are two nonnegative solution of (3). If $P\left(\tilde{u}_{1}(\cdot, t)\right)=P\left(\tilde{u}_{2}(\cdot, t)\right)$ for $0<t<T$, then $\tilde{u}_{1}(\xi, t)=\tilde{u}_{2}(\xi, t)$. Without loss of generality, we assume that

$$
\begin{cases}P\left(\tilde{u}_{1}(\cdot, t)\right)=P\left(\tilde{u}_{2}(\cdot, t)\right), & \text { for } 0 \leqslant t \leqslant t_{0}, \\ P\left(\tilde{u}_{1}(\cdot, t)\right)>P\left(\tilde{u}_{2}(\cdot, t)\right), & \text { for } t_{0}<t \leqslant t_{1},\end{cases}
$$

\section{References}

[1] J. Crank. Free and Moving Boundary Problems. Mathematics of Computation, 46 (174): 429-500, 1986.

[2] Purlis Emmanuel and V. O. Salvadori. Bread baking as a moving boundary problem. Part 1: Mathematical modelling. Journal of Food Engineering, 91 (3): 428-433, 2009.

[3] H. Chen, W. Lv and S. Wu. A free boundary problem for a class of parabolic type chemotaxis model. Kinetic and Related Models, 8 (4): 667-684, 2018.

[4] Pavel Drbek and J. Milota. Methods of Nonlinear Analysis. Automatic Control IEEE Transactions on, 17 (6): 848-849, 1973.

where $0 \leq t_{0}<t_{1} \leq T$. We have

$$
F\left(h(t) \xi, t, \widetilde{u_{1}}, P\left(\widetilde{u_{1}}(\cdot, t)\right)\right) \leq F\left(h(t) \xi, t, \widetilde{u_{1}}, P\left(\widetilde{u_{2}}(\cdot, t)\right)\right)
$$

and

$$
F\left(h(t) \xi, t, \widetilde{u_{2}}, P\left(\widetilde{u_{2}}(\cdot, t)\right)\right) \geq F\left(h(t) \xi, t, \widetilde{u_{2}}, P\left(\widetilde{u_{1}}(\cdot, t)\right)\right)
$$

by $(\mathrm{C} 4)$. Then $\tilde{u}_{1}(\xi, t)$ and $\tilde{u}_{2}(\xi, t)$ are a lower and an upper solution of (3) on $D_{t_{1}}$, respectively. By comparison

principle, we get $\tilde{u}_{1}(\xi, t)<\tilde{u}_{2}(\xi, t)$ a.e. in $D_{t_{1}}$ and hence $P\left(\tilde{u}_{1}(\cdot, t)\right) \leq P\left(\tilde{u}_{2}(\cdot, t)\right)$ for $0 \leq t \leq t_{1}$, which is a contradiction.

\section{Acknowledgements}

The authors of this paper would like to thank the referee for the comments and helpful suggestions.

[5] G. S. Ladde, V. Lakshmikantham, and A. S. Vatsala. Monotone Iterative Techniques for Nonlinear Differential Equations, Pitman, Boston, 1985.

[6] C. V. Pao. Nonlinear Parabolic and Elliptic Equations, Plenum Press, New York, 1992.

[7] A. S. Ackleh and K. Deng. Monotone method for nonlinear nonlocal hyperbolic problems. Electronic Journal of Differential Equations, Conference (10): 11-22, 2003.

[8] A. S. Ackleh. Modeling biological process in aggregation of phytoplankton, PH. D. Thesis, University of Tennessee, Knoxville, TN, 1993.

[9] D. P. Aikman. Modeling of growth and competition in plant monocultures. In Individual-Based Models and Approaches in Ecology, Edited by D. L. Deangelis and L. J. Gross, Chapman and Hall, London, pages 472-491, 1992.

[10] E. D. Ford and K. A. Sorrensen. Theory and models of inter-plant competetion as a spatial process. In IndividualBased Models and Approaches in Ecology, Edited by D. L. Deangelis and L. J. Gross, Chapman and Hall, London, pages 363-407, 1992. 
[11] A. S. Ackleh and K. Deng. Monotone scheme for nonlinear first-order hyperbolic initial-boundary value problems. Applied Mathematics Letters, 13 (5): 111-119, 2000.

[12] H. Brezis. Functional analysis, Sobolev spaces and partial differential equations. Springer Science and Business Media, 2010 .

[13] W. Lv and S. Wu. A free boundary problem for a class of nonlinear nonautonomous size-structured population model. Applicable Analysis, 97 (16): 2852-2864, 2018.

[14] A. S. Ackleh and K. Deng. Existence-uniqueness of solutions for a nonlinear nonautonomous size-structured population model: an upper-lower solution approach. Canadian Appled Mathematics Quarterly, 8: 1-15, 2000.

[15] H. T. Banks and K. Kunisch. Estimation techniques for distributed parameter systems. Estimation Techniques for Distributed Parameter Systems. Birkh"auser Boston, 1989.

[16] A. Calsina and J. Saldana. A model of physiologically structured population dynamics with a nonlinear individual growth rate. Journal of Mathematical Biology, 33 (4): 335364, 1995. 